Emily Hume1, Matthew Armstrong', James Manifield@1, Laura McNeillie², Francesca Chambers², Lynsey Wakenshaw ${ }^{2}$, Graham Burns ${ }^{3}$, Karen Heslop Marshall ${ }^{3}{ }^{3}$, loannis Vogiatzis ${ }^{1}$

emily.c.hume@northumbria.ac.uk

@emilyhume

Editorial

\title{
Impact of COVID-19 shielding on physical activity and quality of life in patients with COPD
}

COVID-19 is a highly infectious disease that can cause severe respiratory illness, particularly in patients with pre-existing lung conditions such as COPD [1]. To prevent infection during the peak of the pandemic, patients considered to be clinically extremely vulnerable were instructed to "shield" at home, resulting in significant restrictions to usual daily activities and social interaction. In the absence of a preventative vaccine, these public health measures are imperative to reduce transmission of the virus. However, despite this positive aspect, there are likely to be harmful repercussions on people's physical and mental health.

The adverse effects of physical inactivity in COPD patients are well known and include worsening of health-related quality of life (HRQoL) [2], along with an increased risk of hospitalisations and mortality [3]. The COVID-19 restrictions have posed challenges for the entire population to stay active, with many turning to online resources for support and guidance. However, some patients with limited access or knowledge of how to use these resources may be disadvantaged and might not benefit. In addition to the instruction to shield, the provision of many respiratory clinical services including pulmonary rehabilitation (PR) were suspended and staff redeployed. These programmes remain imperative for those with chronic lung disease, and the demand is now accompanied by an emerging call for rehabilitation/recovery programmes for COVID19 patients [4]. A wealth of evidence highlights the use of PR for improving exercise capacity, HRQoL and symptoms. PR also promotes adherence to health enhancing behaviours, facilitating the translation of enhanced exercise capacity to greater participation in physical activities [5]. However, upon completion, these benefits tend to diminish over the first 6-12 months if no on-going exercise is performed [6]. The COVID-19 shielding period could, therefore, have potentially exacerbated the loss of health benefits attained by patients recently completing a PR programme. Studies employing maintenance interventions in the form of supervised and technology assisted exercise have shown some promise in maintaining PR benefits [7].

We followed up a cohort of 10 COPD patients (mean \pm SD forced expiratory volume in $1 \mathrm{~s}\left(\mathrm{FEV}_{1}\right)$ : $55 \pm 23 \%$ predicted), who completed an 8 -week PR course between January and March 2020 in the North East of England. Physical activity was measured using accelerometry (Actigraph wGT3X; Actigraph, Pensacola, FL, USA) and the Clinical Visit of Proactive Physical Activity in COPD (C-PPAC) instrument [8] in the week preceding PR, the week following completion of PR and for a week 3 months following completion of PR during the shielding period (April to July 2020). In addition, assessment of HRQoL (COPD Assessment Test (CAT) and Clinical COPD Questionnaire (CCQ)) and psychological wellbeing (Hospital Anxiety and Depression Scale (HADS)) was undertaken. Patients were first contacted by telephone, then an accelerometer and the questionnaires were sent to them in the post. These patients had

@ERSpublications

In response to \#COVID19, healthcare professionals should scale up virtual consultations for assessing core patient-reported outcomes and providing home-based rehabilitation programmes \#COPD https://bit.ly/30gQEpG

Cite as: Hume E, Armstrong M, Manifield J, et al. Impact of COVID-19 shielding on physical activity and quality of life in patients with COPD. Breathe 2020; 16: 200231. 

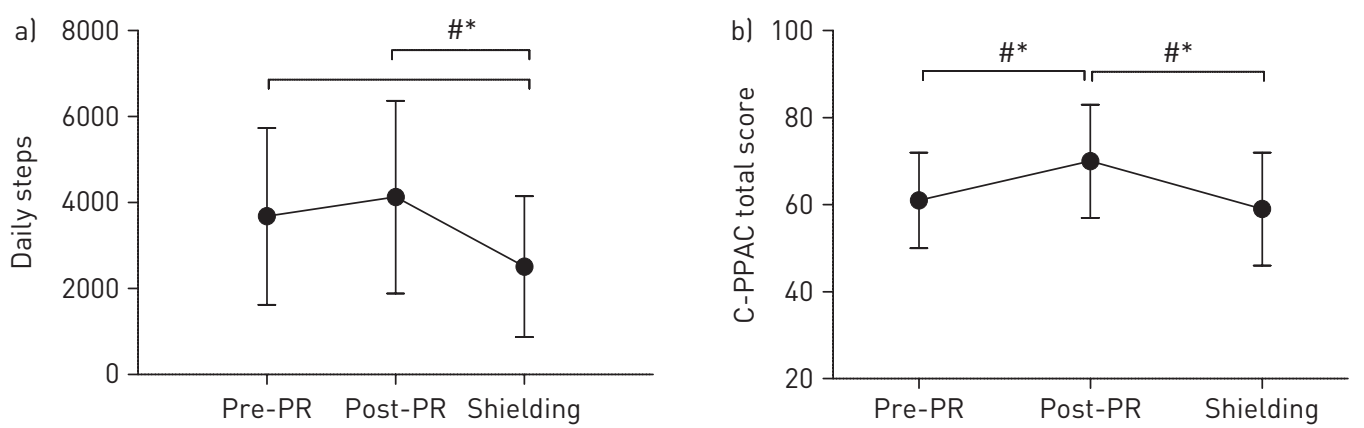

Figure 1 a) Daily steps and b) C-PPAC scores pre-PR, post-PR and during shielding. Data are presented as mean $\pm S D$. *: significant difference between time points, $p<0.05$; : clinically meaningful difference between time points.

been previously enrolled in a study (ClinicalTrials. gov identifier: NCT03749655) investigating the inclusion of physical activity promotion alongside PR and were therefore familiar with the assessment procedures and willing to be monitored.

The results showed a significant and clinically meaningful (>600-1100 steps per day [9]) decrease in daily steps from post-PR to shielding, as well as pre-PR to shielding (figure 1a). The deterioration in daily steps was also accompanied by a significant and clinically meaningful (4 points [8]) worsening in the C-PPAC score from post-PR to shielding (figure $1 \mathrm{~b}$ ), demonstrating not only a reduction in the amount of activity undertaken, but also an increase in the perceived difficulty of conducting physical activity.

A previous multicentre trial involving 157 COPD patients, showed a downward trajectory of physical activity over time, evidenced through a $16 \%$ and $18 \%$ decrease in daily steps over 6 months and a year, respectively [10]. It is clear from our preliminary data, that "shielding" has further exacerbated physical activity decline, shown through a $39 \%$ reduction in daily steps from post-PR to shielding (3 months). The deterioration ( $-1620 \pm 1573$ steps per day) also exceeds that previously exhibited over the year following PR of 600-1000 steps $[11,12]$. Furthermore, there was a $32 \%$ decline from pre-PR to shielding. This worsening below pre-rehabilitation suggests that not only could some of the benefits attained during PR be lost, but also further deconditioning is likely to have occurred, potentially resulting in worsened longterm disease-related outcomes and an increased risk of comorbidities. Additionally, the decline is significantly higher than that seen in the general UK population (-7.8\%) during the lockdown period [13]. This is partly due to the total home confinement of shielding compared with partial confinement for non-vulnerable individuals, as other countries with more stringent restrictions had a greater decline. However, shielding has probably exacerbated the vicious cycle of declining physical activity, leading to increased symptoms and further activity avoidance, which is already evident in COPD patients.

Physical inactivity and deconditioning can often lead to worsening symptoms (breathlessness and leg discomfort), and subsequent worsening of physical functioning and HRQoL [14]. The data collected from this cohort showed a decline in CCQ scores from post-PR to shielding which exceeded clinically meaningful margins ( \pm 0.4 points) for both functional ( +0.5 points) and mental domains (+0.7 points) (figure 2 ). This indicates that shielding caused patients to feel increasingly limited when conducting daily physical activities, which is accompanied with heightened feelings of depression and concerns about breathing. In the general population, the strong link between social isolation and anxiety, depression and cognitive decline is well established [15]. It is perhaps surprising that no statistically or clinically meaningful changes in anxiety or depression (HADS) and health-related quality of life (CAT scores) were seen in this follow-up. It is possible that patients initially felt safer whilst shielding; however, it will be interesting to follow these patients longitudinally to observe if any changes ensue as the uncertainty of the COVID-19 pandemic continues.

\section{Clinical implications}

The findings from this follow-up confirm that the shielding period has caused a significant deterioration in patients' physical activity within a short time period of 3 months. It is important to consider that this data reflects patients who have recently undergone PR; therefore, it is likely that the effects of shielding are worse for the remaining COPD population who have not recently participated in PR. Due to the well-established association between physical inactivity and adverse clinical outcomes, focus on supporting patients to selfmanage their condition and remain physically active at home is imperative. This could be done by using tools, such as tablet computers, pedometers, pulse oximeters and exercise diaries, while providing remote specialist support (e.g. over the telephone) to increase patient confidence to exercise at home independently [16].

The pandemic has increased the urgency of developing novel ways of delivering PR programmes and supporting patients remotely. Clinicians continue to explore alternative modes of remote PR delivery and maintenance, including home-based 

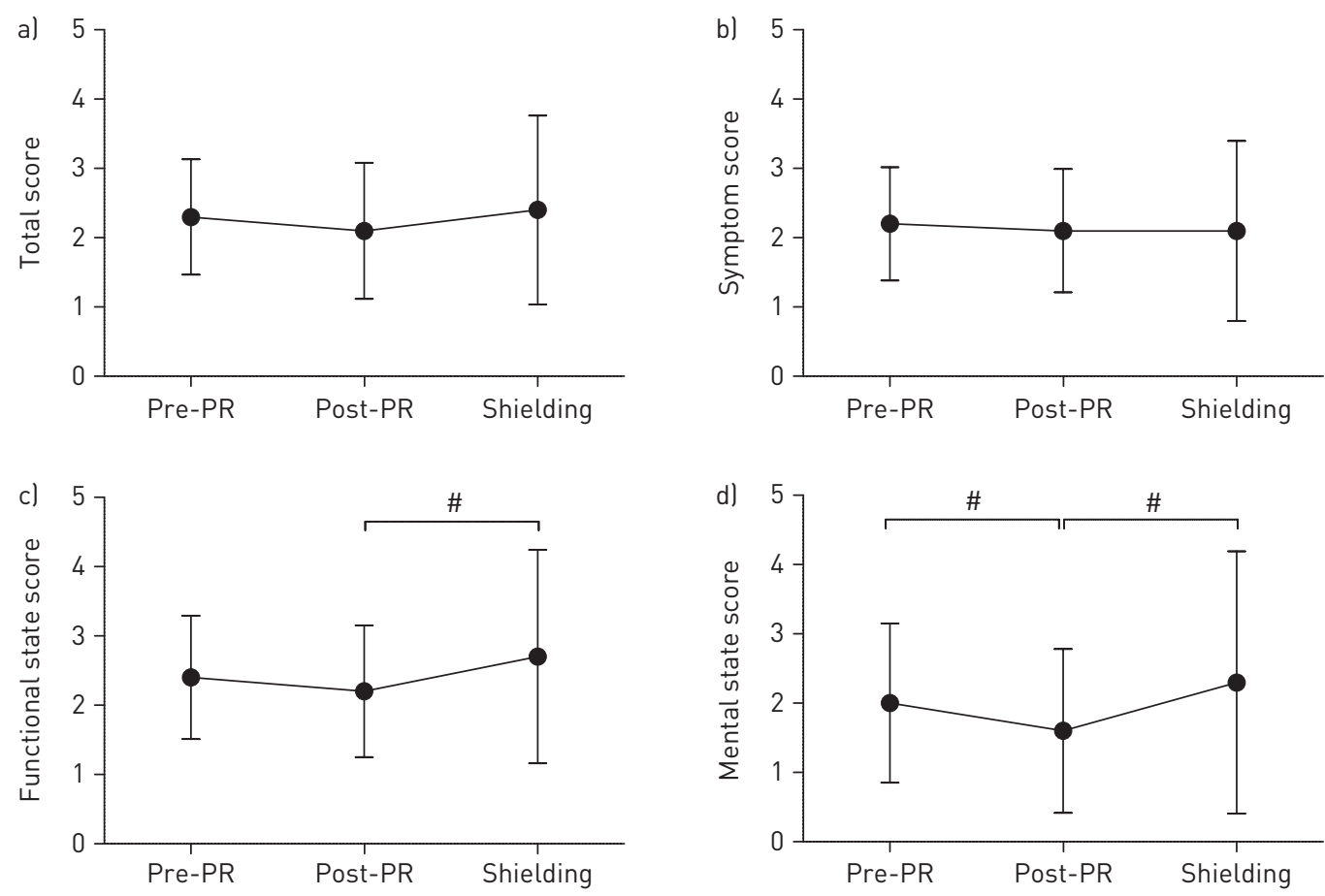

Figure 2 a) CCQ total score, b) symptom score, c) functional state score and d) mental state score pre-PR, post-PR and during shielding. Data are presented as mean $\pm S D$. \#: clinically meaningful difference between time points.

minimally supervised rehabilitation, internet-based programmes, pedometer-based programmes, yoga and t'ai chi exercise. Indeed, virtual consultations have emerged as a consequence of the pandemic, replacing face-to-face consultations. These have been found to be largely acceptable by both patients and clinicians [17]. In the majority of cases, virtual consultations are taking place over the telephone but there is also the possibility for video consultations, when the patient has access to the internet and can use a tablet computer or smart phone. Video consultations may be conducted over communication platforms (e.g. Microsoft Teams or Zoom) or preferably other secure platforms hosted by the National Health Authorities (such as "Attend Anywhere" in the UK).

Virtual consultations should aim to provide patients with a tailored programme at home including: 1) an educational component on managing the disease; 2) psychological support; 3) nutritional support; 4) advice to be physically active indoors and outdoors (if in accordance with public health guidance); 5) access to resource packs for improving well-being; and 6) home diaries for patients to record their progress and symptoms that can be reviewed regularly by healthcare professionals. Where resources are limited, patients may be directed to established sources such as the British Lung Foundation or Lung Foundation Australia with access to specifically designed exercise videos, or handbooks for those without internet access $[18,19]$. Safety guidance for remotely supervised interventions provided by national respiratory societies [20] should be followed closely to ensure patient safety and correct prescription of exercise. The preliminary data from this follow-up indicates that the instruction to shield in vulnerable, clinical populations might have been a double-edged sword, and clinicians should be wary of long-term detrimental effects when returning to patient treatment.

\section{Affiliations}

Emily Hume', Matthew Armstrong ${ }^{1}$, James Manifield D1, Laura McNeillie², Francesca Chambers ${ }^{2}$, Lynsey Wakenshaw $^{2}$, Graham Burns ${ }^{3}$, Karen Heslop Marshall (D) ${ }^{3}$, loannis Vogiatzis ${ }^{1}$

${ }^{1}$ Dept of Sport, Exercise and Rehabilitation, Faculty of Health \& Life Sciences, Northumbria University Newcastle, Newcastle, UK. .'Physiotherapy Dept, The Newcastle upon Tyne Hospitals NHS Foundation Trust, Newcastle, UK. .${ }^{3}$ Chest Clinic, The Newcastle upon Tyne Hospitals NHS Foundation Trust, Newcastle, UK. .

\section{Conflict of interest:}

None declared 


\section{References}

1. Alqahtani JS, Oyelade T, Aldhahir AM, et al. Prevalence, severity and mortality associated with copd and smoking in patients with COVID-19: a rapid systematic review and meta-analysis. PLoS One 2020; 15: e0233147-e.

2. Esteban C, Quintana JM, Aburto M, et al. Impact of changes in physical activity on health-related quality of life among patients with COPD. Eur Respir J 2010; 36: 292-300.

3. Garcia-Aymerich J, Lange P, Benet M, et al. Regular physical activity reduces hospital admission and mortality in chronic obstructive pulmonary disease: a population based cohort study. Thorax 2006; 61: 772-778.

4. Spruit MA, Holland AE, Singh SJ, et al. COVID-19: interim guidance on rehabilitation in the hospital and post-hospital phase from a European Respiratory Society and American Thoracic Society-coordinated International Task Force. Eur Respir J 2020; 56: 2002197.

5. Spruit MA, Pitta F, McAuley E, et al. Pulmonary rehabilitation and physical activity in patients with chronic obstructive pulmonary disease. Am J Respir Crit Care Med 2015; 192: 924-933.

6. Spruit MA, Singh SJ. Maintenance programs after pulmonary rehabilitation: how may we advance this field? Chest 2013; 144: 1091-1093

7. Spencer LM, McKeough ZJ. Maintaining the benefits following pulmonary rehabilitation: achievable or not? Respirology 2019; 24: 909-915.

8. Gimeno-Santos E, Raste Y, Demeyer H, et al. The PROactive instruments to measure physical activity in patients with chronic obstructive pulmonary disease. Eur Respir J 2015; 46: 988-1000.

9. Demeyer H, Burtin C, Hornikx M, et al. The minimal important difference in physical activity in patients with COPD. PLoS One 2016; 11: e0154587-e.

10. Boutou AK, Raste $\mathrm{Y}$, Demeyer $\mathrm{H}$, et al. Progression of physical inactivity in COPD patients: the effect of time and climate conditions - a multicenter prospective cohort study. Int J Chron Obstruct Pulmon Dis 2019; 14: 1979-1992.
11. Holland AE, Mahal A, Hill CJ, et al. Home-based rehabilitation for COPD using minimal resources: a randomised, controlled equivalence trial. Thorax 2017; 72: 57-65.

12. Cruz J, Brooks D, Marques A. Walk2Bactive: A randomised controlled trial of a physical activity-focused behavioural intervention beyond pulmonary rehabilitation in chronic obstructive pulmonary disease. Chron Respir Dis 2016 13: $57-66$.

13. Pépin JL, Bruno RM, Yang RY, et al. Wearable activity trackers for monitoring adherence to home confinement during the COVID-19 pandemic worldwide: data aggregation and analysis. J Med Internet Res 2020; 22: e19787.

14. Troosters T, van der Molen T, Polkey M, et al. Improving physical activity in COPD: towards a new paradigm. Respir Res 2013; $14: 115$

15. Webb L. COVID-19 lockdown: A perfect storm for olde people's mental health. J Psychiatr Ment Health Nurs 2020; in press [https://doi.org/10.1111/jpm.12644].

16. Vasilopoulou M, Papaioannou Al, Kaltsakas G, et al. Home-based maintenance tele-rehabilitation reduces the risk for acute exacerbations of COPD, hospitalisations and emergency department visits. Eur Respir J 2017; 49; 1602129.

17. Gilbert AW, Billany JCT, Adam R, et al. Rapid implementation of virtual clinics due to COVID-19: report and early evaluation of a quality improvement initiative. BMJ Open Qual 2020; 9: e000985.

18. British Lung Foundation. Stay active and stay well. 2020. www. blf.org.uk/support-for-you/keep-active/exercise-video

19. Lung Foundation Australia. Healthy habits. 2020. www. lungfoundation.com.au/lung-health/protecting-your-lungs/ coronavirus-disease-covid-19/physical-health/

20. Singh S, Bolton C, Nolan C, et al. BTS Guidance for pulmonary rehabilitation - Reopening services for the 'business as usual' participants. 2020. Available from: www. brit-thoracic.org.uk/about-us/covid-19-resumption-andcontinuation-of-respiratory-services/ 